\title{
Wrinkling of the eye in hybrids between Drosophila virilis and Drosophila lummei is caused by interaction of maternal and zygotic genes
}

\author{
ERJA HEIKKINEN \\ Department of Genetics, University of Oulu, SF 90570 Oulu, Finland
}

\begin{abstract}
Hybrids of Drosophila virilis females and Drosophila lummei males have visible developmental anomalies in external adult structures. Reciprocal hybrids are normal, and the anomalies are not found in other interspecific $F_{1}$ hybrids of the virilis subgroup. Antibiotic treatments with ampicillin, streptomycin and tetracycline did not cure the syndrome. The genetic basis of the wrinkling of the compound eye was analysed in detail. Both male and female $\mathrm{F}_{1}$ progeny of $D$. virilis mothers were affected, indicating that wrinkling of eyes is maternally determined. $F_{1}$ hybrid females produced healthy progeny. Backcross hybrid females $\left(D\right.$. virilis $\left.\times \mathrm{F}_{1}\right)$ with 16 different combinations of autosomes, either heterozygous or homozygous for $D$. virilis, were crossed with $D$. lummei males. It was found that homozygous $D$. virilis chromosomes 2 and 5 together were necessary to induce the maternal effect. The hybrid zygote responded to the maternal effect when the chromosomes 2, 4 and/or 5 were heterozygous. Elimination of the small sixth (dot) chromosome of D. lummei from the hybrids was correlated with the wrinkling of eyes, but was not the cause, as the two phenomena had a different genetic basis and temperature response. Furthermore, the eyes were wrinkled in flies which presumably had had no $D$. lummei dot chromosome to lose.
\end{abstract}

Keywords: evolution, postzygotic isolation, speciation.

\section{Introduction}

When Drosophila virilis females and Drosophila lummei males are crossed, the majority of the first hybrid generation adult flies suffer from external structural anomalies. This has already been noted by Sokolov (1948, 1959). Recently, Orr (1990) reexamined the phenomenon. He confirmed the maternal origin of the anomalies and excluded any effect of the $\mathrm{X}$ chromosome, which usually plays a large role in the postzygotic isolation mechanisms (Coyne \& Orr, 1989). Sub-lethal morphological anomalies are not rare in species hybrids. Sturtevant (1920) described them in his first paper concerning interspecific hybridization in Drosophila. He studied hybrids between $D$. melanogaster and $D$. simulans, which often had abnormally banded abdomens, broken or missing crossveins and certain bristles absent. Anomalies were obtained in both reciprocals. Coyne (1985) re-analysed anomalies in hybrids of $D$. simulans and D. melanogaster, and also studied combinations with $D$. mauritiana. Weisbrot (1963) reported that in hybrids between $D$. pseudo- obscura females and $D$. persimilis males, 0.2 per cent of the progeny had abnormal tergites.

In this paper the anomalies of $D$. virilis $\times D$. lummei hybrids and their genetic basis are investigated. $D$. virilis and $D$. lummei are weakly isolated, geographically allopatric, sibling species. The genetics and biology of the Drosophila virilis group is described in detail by Throckmorton (1982).

\section{Materials and methods}

\section{Flies}

Two marker stocks of $D$. virilis were used. Stock 126 has the major autosomes recessively marked: $b ; g p ; c d ; p e$. The map positions (Alexander, 1976) are: broken ( $b$ 2-188.0), gapped ( $g p$ 3-118.0), cardinal ( $c d$ 4-32.2), peach (pe 5-203). D. virilis marker stock 162 was $b ; g p ; c d ; p e ; G p g l$ (Gap, Gp 6-0.4, glossy, gl 6-1.0). The stocks 126 and 162 were from the Institute of Developmental Biology, USSR Academy of Sciences, Moscow. For control crosses, the wild type $D$. virilis 
stock 1422 was used (Groeningen, The Netherlands, 1976).

In genetic analyses extending to the second and third generation, D. lummei stock $1101 \mathrm{~S}$ was used (Överkalix, Sweden, 1970). In addition, wild caught $D$. lummei males from Kemi, Finland were crossed individually to single females from the $D$. virilis marker stock 126.

$\mathrm{F}_{1}$ hybrids were inspected from all possible combinations between at least two stocks of each taxa $D$. virilis, $D$. lummei, $D$. novamexicana, $D$. americana americana and $D$. americana texana (The National Drosophila Species Resource Center, Bowling Green, Ohio, USA).

The stocks were reared on malt medium (Lakovaara, 1969) at $19^{\circ} \mathrm{C}$ under continuous illumination and 70 per cent relative humidity.

\section{Antibiotic treatments}

To test whether the wrinkling of the compound eye was caused by some symbiotic micro-organism, culture medium was supplemented to a final concentration of 0.03 per cent with ampicillin, streptomycin, tetracycline, or both ampicillin and streptomycin (both 0.03 per cent), following Hoffmann et al. (1986). Tetracycline bottles were lined with aluminium foil, to keep them dark. Sodium chloride, up to a final concentration of 0.67 per cent, was used as a control.

$D$. virilis marker stock 126 and wild type $D$. lummei $1101 \mathrm{~S}$ were reared on the antibiotics supplemented medium in $\mathrm{P}$ and $\mathrm{F}_{1}$ hybrid generations, and scored for the wrinkled eye syndrome.

\section{Statistical analysis}

In testing the proportions of damaged flies, a standard $G$-test, $G$-test for independence, and Student's $t$-test were used (Sokal \& Rohlf, 1981). A log-linear model and the $G$-statistic were used to analyse the progeny families of wild-caught flies (Sokal \& Rohlf, 1981). The contributions of chromosomes and their interactions on the expression of the eye syndrome were treated as a modification of the analysis of factorial experiments (Snedecor \& Cochran, 1967; Lumme \& Heikkinen, 1990).

\section{Results}

\section{External structural anomalies in the $F_{1}$ generation}

External structure anomalies are illustrated in Fig. 1.

Abdominal cuticula. All the emerged $\mathrm{F}_{1}$ adults from crossing $D$. virilis females to $D$. lummei males had abnormalities in the abdominal chitinization, i.e. in sternites (Fig. 1f) and tergites (Fig. 1h). The eighth tergite, derived from the genital disc, was invariably normal. Tergites 1-7 are derived from the abdominal histoblasts. Tergites 2 and 3 were most often and most badly misshapen, and their bristle patterns irregular. The joining of hemitergites had frequently failed (Fig. 1h). This type of developmental disorder was rare in pure stocks, and still less frequent in the reciprocal cross $D$. lummei $\times D$. virilis.

Other anomalies were less frequent. Out of 2975 emerged hybrids of $D$. virilis $\times D$. lummei, 2430 had no other external anomalies except abnormalities in abdominal cuticula. The frequency and pairwise cooccurrence of anomalies in eyes, legs, wings, antennae and halteres are presented in Table 1 . In a few cases, three or four of these organs were affected in one individual. Data in Table 1 are pooled over temperatures and sexes.

Wrinkling of the eye. In total, 8.7 per cent of the 2975 $F_{1}$ flies from the cross $D$. virilis $\times D$. lummei had wrinkled eyes (Fig. 1b-d). As a control, 2719 pure $D$. virilis were studied; nine of them $(0.33$ per cent $)$ had wrinkled eyes. Out of the 1118 flies studied from the reciprocal cross, only one had the wrinkled eye syndrome.

There were significantly more wrinkled eyes in the flies cultured at $25^{\circ} \mathrm{C}$ than at $17^{\circ} \mathrm{C}(10.1$ per cent, $n=1540$ vs. 6.5 per cent, $n=1435 ; t=3.5, P<0.001)$.

Males and females were almost equally affected. There were no significant differences in the numbers of affected males and females (at $17^{\circ} \mathrm{C}$ : males 5.1 per cent, $n=650$, females 7.6 per cent, $n=785, t=1.9$, $P>0.05$; and at $25^{\circ} \mathrm{C}$ : males 12.0 per cent, $n=664$, females 9.1 per cent, $n=876, t=1.9, P>0.05$ ).

Only the right eye was wrinkled in 123 cases, only the left one in 130 cases. Both eyes were wrinkled in six individuals, when the expected number was 5.4 $\left(G_{(1)}=0.090, P>0.75\right)$. The left and the right side of an individual thus seemed to be independent.

Misshapen legs. Legs were considered as abnormal, if any of the six was considerably shorter than its pair; sometimes a leg was missing, and one fly with an extra leg was observed. The frequency of leg abnormalities was 5.4 per cent. Leg anomalies occurred significantly more often in flies that also had misshapen eyes $(25$ observed, 13.9 expected, $\left.G_{(1)}=8.518, P<0.01\right)$.

Wing anomalies. Wings were missing, or sometimes comprised of two blades. Small vein defects were not counted. Altogether, 1.9 per cent of the flies were scored with abnormal wings. 

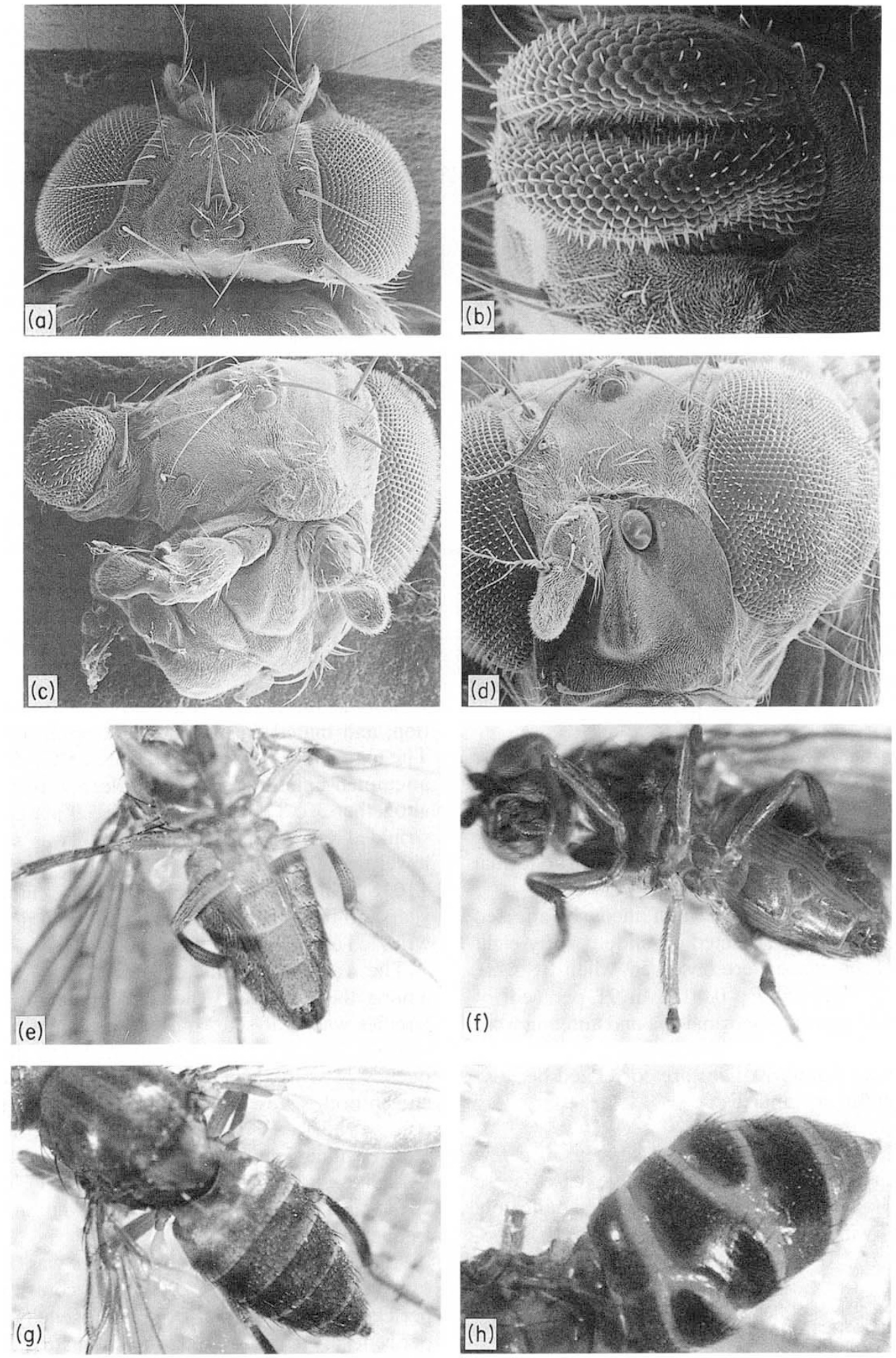

Fig. 1 Morphological anomalies in hybrids between D. virilis females and D. lummei males. (a) Compound eyes of normal D. lummei. (b) Mildly wrinkled eye in $F_{1}$ hybrid. (c and d). Eye-antennae anomalies in $F_{1}$ hybrids. (e) Sternites of a normal D. lummei male. (f) $\mathrm{F}_{1}$ male with abnormal sternites. (g) Tergites of a normal D. lummei female. (h) Abnormally joined hemitergites in an $F_{1}$ female. 
Table 1 Pairwise co-occurrence of abnormalities in the first hybrid generation between $D$. virilis females and $D$. lummei males. Along the diagonal are the numbers of flies with only one organ damaged. The off-diagonal cells show the numbers of flies with anomalies that occur together. Data are pooled over temperatures and sexes, $n=2975$

\begin{tabular}{lcccccr}
\hline & Eye & Leg & Wing & Antenna & Halter & Total \\
\hline Eye & 162 & $25^{* *}$ & $12^{* *}$ & $58^{* * *}$ & 2 & 259 \\
Leg & & 126 & $8^{* *}$ & 1 & 0 & 160 \\
Wing & & & 33 & 1 & 0 & 54 \\
Antenna & & & 7 & 0 & 67 \\
Halter & & & & 3 & 5 \\
Healthy & & & & & 2430 \\
\hline
\end{tabular}

${ }^{*} P<0.05,{ }^{* *} P<0.01,{ }^{* * *} P<0.001$. Test of independence using $G$-Statistic, d.f. $=1$. All four significant deviations are positive, i.e. the anomalies occurred together more often than might be expected at random.

The eye and the wing were defective simultaneously more frequently than might be expected at random $(12$ flies, 4.7 expected, $\left.G_{(1)}=9.262, P<0.01\right)$. In eight flies, leg and wing were simultaneously affected and this is significantly more than the random expectation $(2.9$ expected, $\left.G_{(1)}=6.723, P<0.01\right)$.

Antennal anomalies. Rudimentary antennae were most common, and sometimes antennae with leg-like appendages (Fig. 1c and d) were found. Two flies with three antennae and one with both antennae missing were recorded. The flies scored with abnormal antennae amounted to 2.3 per cent.

Antennae and eyes were simultaneously affected much more often than expected at random. Fifty-eight cases of co-occurrence were observed, when 5.8 were expected $\left(G_{(1)}=244.5, P \ll 0.001\right)$. In 91 per cent of the 58 cases observed, abnormal eye and antenna were on the same side of the fly. This demonstrates that the eye and antennal abnormalities are correlated because of their common imaginal disc.

Halteres. Halteres were seldom misshapen; in a few cases they resembled wings. Only five cases were observed. Haltere anomalies were not significantly correlated with other anomalies $\left(G_{(1)}=4.098\right.$, $P<0.05)$.

\section{Effect of antibiotics on the wrinkling of the eyes}

The frequencies of hybrids with wrinkled eyes from the vials containing the antibiotics are given in Table 2 . In comparison to the control, treatment with tetracycline, ampicillin, or streptomycin decreased the frequency of
Table 2 Proportion of $F_{1}$ hybrid flies with wrinkled eyes $(\%$, $n$ within brackets), when parental and $F_{1}$ generations had been reared on antibiotics. Student's $t$-test compares the effect of each treatment with the control

\begin{tabular}{lllll}
\hline Drug & $\%$ & $n$ & $t$ & \\
\hline Control $(0.67 \% \mathrm{NaCl})$ & 7.8 & $(217)$ & & \\
Ampicillin $(0.03 \%)$ & 3.5 & $(288)$ & 2.126 & $P<0.05$ \\
Streptomycin $(0.03 \%)$ & 3.5 & $(764)$ & 2.711 & $P<0.01$ \\
Tetracycline $(0.03 \%)$ & 2.8 & $(145)$ & 1.995 & $P<0.05$ \\
Ampicillin + streptomycin & 5.2 & $(862)$ & 1.474 & NS \\
\hline
\end{tabular}

wrinkled eyes significantly. However, the combined effect of ampicillin and streptomycin was not significant.

Quantitative data on the defects of the abdominal chitinization were not collected but very obviously they were not cured by antibiotic treatment. Moreover, all other external structural anomalies were also observed in the treated flies.

\section{Wrinkled eyes in the progenies of wild-collected D. lummei males}

D. lummei males were collected from the wild population, and mated pairwise with $D$. virilis 126 females. The progenies were reared at $25^{\circ} \mathrm{C}$. Out of 51 attempted families, 35 were fertile, and they produced altogether 3088 hybrids, of which 5.9 per cent had the wrinkled eye syndrome. This value was significantly lower than that obtained with the males from the stock $1101 \mathrm{~S}$, which had been in laboratory culture for 20 years $\left(1540\right.$ hybrids reared at $25^{\circ} \mathrm{C}, 9.76$ per cent with wrinkled eye, $t=4.866, P<0.001)$.

The variation in the frequency of wrinkled eye among the males and the females in the 35 full-sib families was analysed as a log-linear model, using $G$ statistic. The null hypothesis tested was that the frequency of wrinkled eyes was the same in all families and in both sexes. The total deviation was significant $\left(G_{(69)}=185.1, \quad P<0.001\right)$. Males were more often affected than females ( 7.8 per cent, $n=1481$ vs. 4.9 per cent, $\left.n=1607, G_{(1)}=8.572, P<0.01\right)$. This difference was 4.6 per cent of the total deviation from even frequencies.

The variation between the families was also significant $\left(G_{(34)}=132.9, P<0.001\right)$ and contributed 71.8 per cent to the deviation from homogeneity. This 71.8 per cent can be considered as an estimate of heritability in the wild population of $D$. lummei and in the marker stock of $D$. virilis.

The family $\times$ sex interaction was not significant $\left(G_{(35)}=43.615, P>0.05\right)$, and the contribution value 
23.6 per cent of the interaction can be considered as an error estimate for the heritability.

\section{Elimination of the $\mathrm{D}$. lummei dot chromosome from the $F_{1}$ hybrids}

According to Sokolov (1959), in hybrids between $D$. virilis females and D. lummei males the sixth chromosome (dot) of $D$. lummei is eliminated during early embryogenesis, which has been confirmed by Sidorova (1974). If it is eliminated in the first mitotic cleavage, the entire hybrid is monosomic for the dot chromosome. The later this elimination occurs, the larger will be the proportion of tissues heterozygous for $D$. lummei/D. virilis dot chromosomes. By using the visible $D$. virilis marker, glossy eye $(g l 6-1.0)$ the elimi nation of the $D$. lummei dot chromosome can be detected on the surface of the compound eye.

$D$. virilis stock $162 b ; g p ; c d ; p e ; G p g l$ females were mated with $D$. lummei $1101 \mathrm{~S}$ males. The eyes of the first hybrid generation were scored for wrinkled eye and glossy eye surface phenotypes. The results are displayed in Table 3. The interdependence of the two phenotypes is strong and highly significant $\left(G_{(2)}=\right.$ $270.4, P \ll 0.001)$.

\section{Response to the maternal effect in the backcross D. virilis $\times F_{1}$}

In order to analyse the inheritance of the wrinkling of the eyes in more detail, reciprocal $F_{1}$ males were backcrossed to the marker stock 126 of $D$. virilis. The progenies were reared at $25^{\circ} \mathrm{C}$.

D. $\quad$ virilis $\times(D . \quad$ virilis $\times D$. lummei $), \quad(\mathrm{b} ; \mathrm{gp} ; \mathrm{cd} ; \mathrm{pe}) \times$ $[(\mathrm{b} ; \mathrm{gp} ; \mathrm{cd} ; \mathrm{pe}) \times 1101 \mathrm{~S}]$

Females from this cross had a conspecific set of sex chromosomes, $\mathrm{X}^{\mathrm{vi}} / \mathrm{X}^{\mathrm{vi}}$. The frequency of wrinkled eyes was 1.9 per cent $(n=1930)$. The sex chromosome set of males was $\mathrm{X}^{\mathrm{vi}} / \mathrm{Y}^{\mathrm{lu}}$, and the frequency of wrinkled eyes 0.9 per cent $(n=1597)$.

D. $\quad$ virilis $\times(D$. lummei $\times D$. virilis $),(\mathrm{b} ; \mathrm{gp} ; \mathrm{cd} ; \mathrm{pe}) \times$ [1101S $\times(b ; g p ; c d ; p e)]$

Table 3 Co-occurrence of wrinkled and glossy eye phenotypes in $D$. virilis $162 \times D$. lummei $1101 \mathrm{~S}$ crosses. The figures are numbers of eyes, not of individuals. The total number is uneven because one female had only one eye

\begin{tabular}{lcc}
\hline Phenotype & Normal & Wrinkled \\
\hline Whole eye glossy & 2 & 46 \\
Mosaic + /glossy & 229 & 25 \\
Eye wild type & 614 & 9 \\
\hline
\end{tabular}

Female progeny had heterospecific $\mathrm{X}$ chromosomes, $\mathrm{X}^{\mathrm{lu}} / \mathrm{X}^{\mathrm{vi}}$. Of these 1.6 per cent had wrinkled eyes $(n=2218)$. The sex chromosomes of males were $\mathrm{X}^{\mathrm{vi}} /$ $\mathrm{Y}^{\mathrm{vi}}$, and the frequency of the eye anomaly 0.4 per cent $(n=1993)$.

Contributions of the zygotic chromosomes on the wrinkling of the eye are given in Fig. 2. The males and the females had different frequencies of the eye anomaly (pooled over two crosses, females 1.74 per cent, $n=4148$ vs. males 0.64 per cent, $n=3590$, $t=4.362, P<0.001$ ).

The two crosses were not different, however, in spite of the qualitative differences in the sex chromosomes of the progenies. The similarity extended to the autosomal phenotypes. Furthermore, the similarity also indicates that the role of the sixth chromosome is not decisive. The two crosses are pooled in Fig. 2, where the role of the four large autosomes is analysed.

The highest proportion of females with wrinkled eyes, 4.2 per cent $(n=569)$, was in the autosomal phenotype class $+;+;+;+$, which resembled $F_{1}$. This
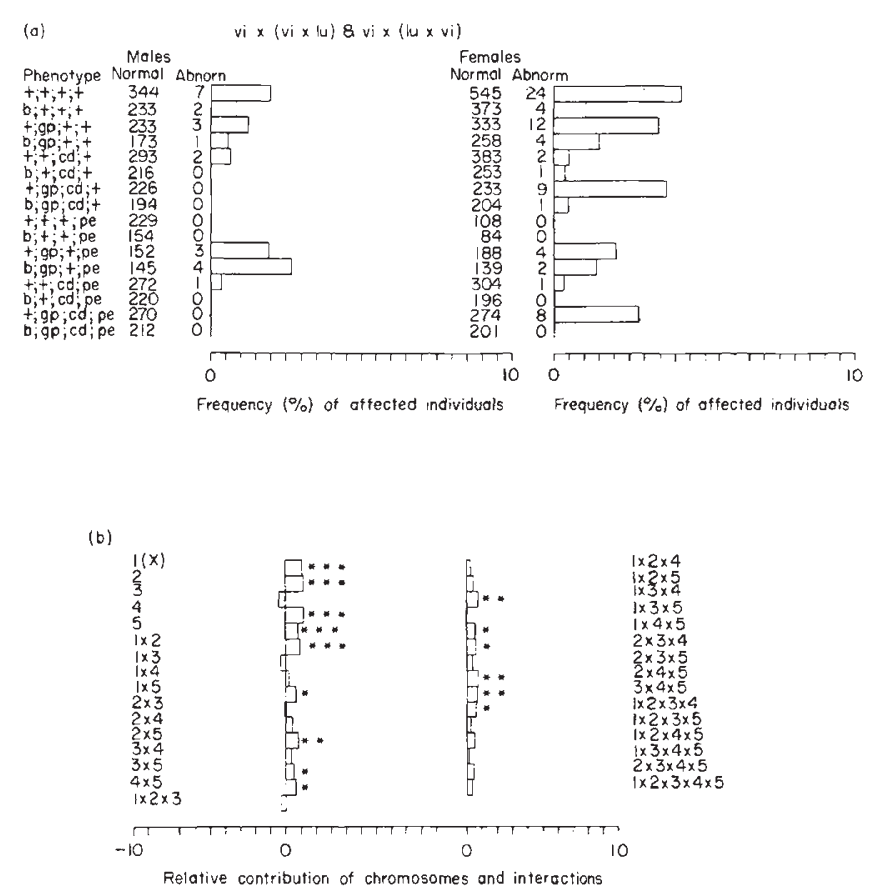

Fig. 2 Contribution of the zygotic chromosomes on the eye wrinkling. (a) Proportions of flies with wrinkled eye in the pooled backcrosses. (b) The analysis of factorial effects of the autosomes 2-5 and sex (1) on the frequency of wrinkled eye. A positive value for the main effect of an autosome means that heterozygous flies had more frequently wrinkled eyes than homozygous (for $D$. virilis chromosome). For primary interactions, a positive value means that flies with a similar origin of respective autosomes are less frequently damaged than those with different. Significance levels of the contributions: ${ }^{*} P<0.05,{ }^{* *} P<0.01,{ }^{* * *} P<0.001$. 
frequency was significantly lower than in the $\mathrm{F}_{1}$ females at $25^{\circ} \mathrm{C}$ (9.1 per cent, $\left.n=876: t=3.5, P<0.001\right)$. It is to be pointed out that the frequency of wrinkled eyes was higher in females carrying $X^{\mathrm{vi}} / \mathrm{X}^{\mathrm{vi}}$ (13 out of 211) than in those exactly like $F_{1}: X^{\mathrm{lu}} / \mathrm{X}^{\mathrm{vi}}(11$ out of 358$)$. Therefore, it seems safe to conclude that the zygotic $X$ chromosomes have no role in eye wrinkling in females.

In males, the largest proportion of abnormals, 2.7 per cent $(n=149)$ was in phenotype class $b ; g p ;+; p e$. In phenotype class $+;+;+;+$, the proportion of affected flies was 2.0 per cent $(n=351)$. This proportion differs significantly from the proportion in $\mathrm{F}_{1}(12.0$ per cent, $n=664: t=5.4, P<0.001) . \mathrm{F}_{1}$ and $\mathrm{BC}_{1}$ $+;+;+;+$ males had the same genotype for chromosomes $2-5 . F_{1}$ males of the affected cross had the sex chromosome set $\mathrm{X}^{\mathrm{vi}} / \mathrm{Y}^{\mathrm{lu}}$. Backcross males had either the same combination (then 4 out of 157 wrinkled) or both sex chromosomes derived from $D$. virilis (three out of 194 wrinkled). This leads to the conclusion that the zygotic sex chromosomes have no role in the wrinkling of eyes in males, either. The difference between males and females has no chromosomal explanation.

Chromosomes 2, 4 and 5, when heterozygous in zygote, increase the incidence of wrinkled eyes. They had significant main effects and also significant primary $(2 \times 5,4 \times 5)$ and secondary interactions $(2 \times 4 \times 5)$. The role of heterozygous chromosome 4 in males was especially clear: 20 males out of 23 having the wrinkled eye syndrome were heterozygous for chromosome 4 $(+/ c d 1.2$ per cent, $n=1683$ vs. $c d / c d 0.2$ per cent, $n=1906, t=3.7, P<0.001$ ).

\section{Localization of the maternal effect on D. virilis chromosomes}

To localize the maternal effect, the second generation backcross hybrid females (at least 10 females of each of the 16 phenotypes) were crossed with $D$. lummei males and the frequency of wrinkled eyes was scored in their progenies reared at $25^{\circ} \mathrm{C}$. Females with genotype $b ; p e /$ $b ;+$ were difficult to breed, and phenotypes $b ; g p ;+;+$ and $b ;+; c d ;+$ produced 5 and 0 flies, respectively, in spite of a much increased effort.

$[D . \quad$ virilis $\times(D . \quad$ virilis $\times D$. lummei $)] \times D$. lummei, $[(\mathrm{b} ; \mathrm{gp} ; \mathrm{cd} ; \mathrm{pe}) \times((\mathrm{b} ; \mathrm{gp} ; \mathrm{cd} ; \mathrm{pe}) \times 1101 \mathrm{~S})] \times 1101 \mathrm{~S}$

The backcross mothers in this cross had conspecific $X$ chromosomes, $\mathrm{X}^{\mathrm{vi}} / \mathrm{X}^{\mathrm{vi}}$. Among their progenies, $1.3 \mathrm{per}$ cent had wrinkled eyes (weighted mean over all phenotypes, even if phenotype frequencies are arbitrary, $n=4823$ ).

$[D . \quad$ virilis $\times(D . \quad$ lummei $\times D . \quad$ virilis $)] \times D$. lummei, $(\{($ b;gp;cd;pe $) \times[1101 \mathrm{~S} \times(\mathrm{b} ; \mathrm{gp} ; \mathrm{cd} ; \mathrm{pe})]\}) \times 1101 \mathrm{~S}$
The mothers in this cross had heterospecific $\mathrm{X}$ chromosomes, $X^{\text {lu }} / \mathrm{X}^{\mathrm{vi}}$. The frequency of wrinkled eyes was 1.1 per cent, $n=3988$. This was not significantly different from the value in the first cross $(t=0.85$, $P \gg 0.1$ ). Not only were the overall means identical, but also the occurrence of wrinkled eyes among the female autosomal phenotypes were similar irrespective of the $\mathrm{X}$ chromosome. It is to be concluded that the female $\mathrm{X}$ chromosomes do not contribute to the maternal effect.

Among the progenies of pooled crosses, the males and the females were equally affected $(t=0.26$, $P>0.05, n$ of males 3974 and $n$ of females 4837).

The proportion of flies with wrinkled eyes in the progenies of females of each of the 16 different backcross phenotypes are given in Fig. 3. The data were pooled over the two crosses (different female $\mathrm{X}$ chromosomes) and the sexes of the progeny.

It is obvious that if the mother was homozygous for the $D$. virilis chromosomes 2 and 5, the progeny contained significantly more flies with wrinkled eyes than otherwise. The proportion of abnormals among the progenies of $b ; p e / b ; p e$ mothers was 6.4 per cent $(n=1353)$, whereas the weighted mean of abnormals among all other phenotypes was 0.2 per cent $(n=7458)$. In fact, out of the 105 handicapped individuals of this generation, 87 were born from $b ; p e / b ; p e$ mothers. The analysis in Fig. $3 \mathrm{~b}$ is based on a comparison of weighted means. If unweighted means are used, the contributions of the second and fifth chromosomes are equal. Chromosome 4 plays no role in the wrinkling of eyes unlike the sixth chromosome of $D$. lummei.

\section{Discussion}

The experiments reported here describe maternally determined external structural anomalies of species hybrids. The case was originally described by Sokolov (1948, 1959).

In the progenies of wild caught $D$. lummei males the total frequency of flies with wrinkled eye was slightly lower than in the progenies of laboratory stock males. However, the trait was frequent enough to imply that the anomaly was not due to strains being kept for a long time in the laboratory ( $>20$ years). The low frequency was due to several pairs not producing handicapped progeny. The experiment revealed that there is genetic variation in $D$. lummei in the response to the maternal effect. This variation is also manifested as variation between D. lummei stocks (data not shown).

During this study, several stocks of $D$. virilis were tested, and there seems to be a consistent maternal effect (data not shown). All other possible hybridizations between the species in the virilis subgroup have 

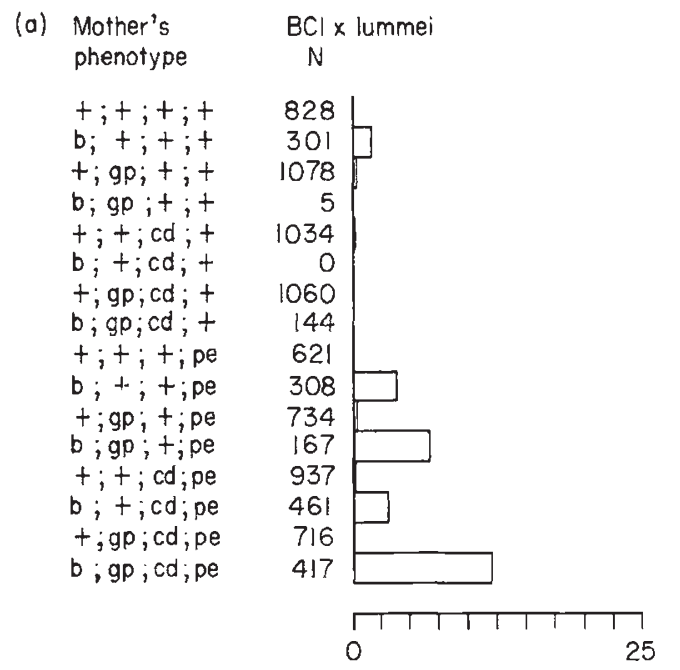

Frequency $(\%)$ of affected individuals

(b) 2

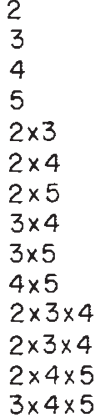

$2 \times 3 \times 4 \times 5$

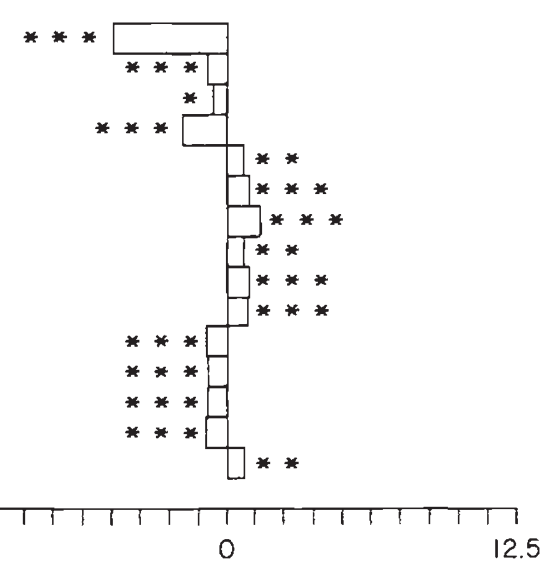

Relative contributions of chromosomes and interactions

Fig. 3 Contributions of the maternal chromosomes on eye wrinkling. (a) The proportions of wrinkled eye individuals in the progenies of females of 16 different backcross phenotypes, crossed to $D$. lummei. Data are pooled over the $\mathrm{X}$ chromosome combinations. (b) The analysis of the data showing the relative autosomal contributions. The main effect is negative when the progenies of females homozygous for $D$. virilis chromosome were more affected than the progenies of heterozygous females. A positive value for a primary interaction means that the frequency of wrinkled eyes was lower in the progenies of females, which have a similar genotype in both chromosomes, than if the genotypes are different. Significance levels of the contributions:

${ }^{*} P<0.05,{ }^{* *} P<0.01,{ }^{* * *} P<0.001$.

also been tested. No other crosses displaying structural anomalies were found.

It has been observed earlier that $D$. virilis $\times D$. lummei $\mathrm{F}_{1}$ hybrids failed to emerge from the pupal case more often than reciprocals, where almost all pupae developed to adult flies (Lumme \& Heikkinen, 1990). The failure to emerge might be connected with external structural anomalies. The morphology of flies dying within the pupal case remains to be studied. Perhaps the abnormalities observed in this study are the least serious fraction of developmental defects.

The anomalies co-occurred in different organs of the same fly more often than expected at random. Simultaneous eye-leg, eye-wing, and leg-wing combinations were significantly more frequent than expected. However, the left and the right eye showed independent anomalies. The strongest correlation was between the compound eye and antenna, which were simultaneously anomalous 10 times as frequently as expected (and at the same side of the fly). The compound eye and antenna develop from the same imaginal disc, and the co-occurrence of defects indicates that the anomaly dates back to the imaginal disc determination.

There were abnormalities both in the anterior and the posterior parts of the body. Some large scale morphological maternal mutations in Drosophila are known, which disturb the global fate map of the egg (Davidson, 1986). The original function of these factors may be in some common cellular function, as in cell formation or multiplication. Some indications of separate maternal control for cephalic and caudalic cell specification are also known, such as bicaudal. Not many mutations with a maternal effect on the early zygote mitosis are known, even in $D$. melanogaster.

\section{The role of the elimination of the microchromosome}

During completion of this study, Orr (1990) published an analysis of the anomalies, concluding that the proximal cause of the defects is the elimination (equal to the absence of the sixth chromosome) of the microchromosome (sixth chromosome) of D. lummei in hybrids. Sokolov's (1959) conclusion was:

'If we look more carefully at the compound eye surface mosaics, which are caused by microchromosome elimination, we see that the process [microchromosome elimination] cannot cause all the anomaly types of eyes detected in the hybrids.'

The resemblance of the two phenomena is striking, and they are closely correlated. However, the results obtained during this study do not confirm this hypothesis. Mitrofanov et al. (1990) have showed that the elimination of the sixth chromosome involves not only a maternal effect, but also some structural and functional specialities of the genome of $D$. lummei, as the dot chromosome is sometimes eliminated from the $D$. lumme $\times D$. virilis hybrids.

The proportion of wrinkled eyes was significantly lower at 17 than at $25^{\circ} \mathrm{C}$. Orr (1990) observed more 
anomalies (all types pooled) at 18 than at $22^{\circ} \mathrm{C}$. The proportion of $\mathrm{F}_{1}$ hybrids with monosomic $D$. virilis dot chromosome is 100 per cent at $17^{\circ} \mathrm{C}$, much more than at $25^{\circ} \mathrm{C}$ (Sidorova, 1974). Evgen'ev et al. (1983), who studied the toromere of $D$. lummei, showed that the elimination of the sixth chromosome of the species increased if the culture temperature decreased and if the sixth chromosome had to replicate in $D$. virilis genetic conditions. If elimination would cause the wrinkling then the proportion of anomalous flies should increase at lower temperature. The temperature dependence thus remains controversial. A more detailed developmental profile is needed.

Orr (1990) crossed different taxa of the virilis subgroup and observed the co-occurrence of the elimination of the sixth (glossy phenotype) and developmental anomalies. $D$. virilis $\times D$. lummei was the only combination where both phenomena were common. This is no strong evidence for a common basis. In the results presented here, the phenotypic correlation between the appearance of the visible marker of the sixth chromosome, glossy, and the wrinkling of the eye in $\mathrm{F}_{1}$ is striking. Correlation between these phenomena does not mean that their relationship is causal. The conclusion is that at the individual phenotypic level, wrinkling occurs much more often in eyes which are haploid for the $D$. virilis sixth chromosome. This analysis will be extended to the genetic level.

\section{Maternal effect is nuclear and autosomal (the second and the fifth chromosome of $\mathrm{D}$. virilis)}

The anomalies were found only in one of the reciprocal crosses and they were equally frequent in males and females, in accordance with Orr's (1990) findings. Because female progenies from both reciprocal crosses carried an identical set of heterozygous $\mathrm{X}$ chromosomes, these observations lead to the assumption of a maternal effect. This could be caused by three different mechanisms: nuclear genes, mitochondrial genes, or endosymbionts.

Barr (1980) showed that the incompatibility of two strains of Culex pipiens is due to a rickettsia-like bacterial symbiote. Hoffmann et al. (1986) and Hoffmann \& Turelli (1988) found that a similar situation existed between some populations of $D$. simulans. The $D$. virilis $\times D$. lummei cross was repeated on antibiotic supplemented medium in order to test the possibility that the anomalies are caused by a symbiotic micro-organism. Both males and females of the parental generation were treated with ampicillin, streptomycin, and tetracycline, from egg to adult, as well as the $F_{1}$.

Although the frequency of individuals with wrinkled eyes decreased following treatment by all the antibiotics, the anomalies were not otherwise cured. The compound effect of ampicillin and streptomycin was not significant. The vials were attacked by different kinds of molds, bacteria and Histiostoma laboratorium mites. In superinfected vials, a large amount of flies died within pupal cases. This probably affected the frequency of handicapped flies by killing a larger proportion of them than normal. Abnormal flies tend to eclose later than normal flies.

Orr (1990) found a higher proportion of externally abnormal hybrids from tetracycline-supplemented vials than from normal vials, but the proportion of eye anomalies also decreased in his experiment.

These results suggest that wrinkling of eyes in hybrids is not caused by a micro-organism but rather by the interactions of maternal and zygotic genes in the hybrids.

The maternal effect of $D$. virilis females was analysed by producing backcross females with different combinations of heterozygous and homozygous $\mathrm{X}$ and large autosomes. To keep the chromosomes intact, $F_{1}$ males were used for backcrossing, and therefore all female genotypes tested carried the mitochondrial genes of $D$. virilis. Among them, the maternal effect was solely caused by the simultaneous presence of the homozygous second and fifth chromosomes of $D$. virilis. In contrast to the reduced viability and fertility of interspecific hybrids, neither the $\mathrm{X}$ chromosome of the mother $(D$. virilis $)$ nor the $\mathrm{X}$ chromosome of the zygote was found to play any significant role. Orr (1990) made crosses which demonstrated that the mitochondrial genes of $D$. virilis do not participate in the induction of zygotic anomalies.

Microchromosome elimination is also determined maternally by nuclear genes (Sokolov, 1959; Orr, 1990). Sidorova (1974) showed that at low temperatures the elimination of the dot chromosome occurs in the first mitotic cleavage. The maternal control of the elimination of the D. lummei dot chromosome was carefully analysed by Mitrofanov \& Sidorova (1979). Their data consisted of 24,709 offspring of backcross females. At $25^{\circ} \mathrm{C}$, only the second chromosome contributed to the maternal effect. Among the progeny of $b / b$ females, 33.7 per cent $(n=7065)$ lose the microchromosome of $D$. lummei, when the proportion was 1.8 per cent in progeny of $b /+(n=10,032)$. At $17^{\circ} \mathrm{C}$, the frequency of elimination was 100 per cent in the progenies of all genotypes carrying the fourth chromosome as homozygous. Among the fourth chromosome heterozygotes, a heterozygous second chromosome was able to partially rescue the microchromosome. When the wrinkling of the eye was clearly controlled by simultaneous homozygous factors on $D$. virilis 
chromosomes 2 and 5 , it is to be concluded that the genetic basis of maternal effect is not identical for the two traits.

\section{Zygotic response is polygenic, autosomal}

The present results indicated that the second, the fourth and the fifth chromosomes of $D$. lummei when heterozygous in the $F_{1}$ facilitate the wrinkling of the eye. Their role is polygenic, with some mild nonadditive interactions (Fig. 2). Thus, the sixth chromosome of $D$. lummei is not alone in responding to the maternal effect.

The similarity of the proportions of the wrinkled eyes in backcrosses to reciprocal $F_{1}$ does not give any new information about the role of the sixth chromosome in the wrinkling of eyes. If in $\mathrm{F}_{1}(D$. virilis $\times D$. lummei) the sixth chromosome of $D$. lummei is already eliminated, the elimination cannot be the reason for wrinkling in $D$. virilis $\times F_{1}$. Elimination is infrequent in the germ-line (Evgen'ev \& Sidorova, 1976). On the other hand, if $\mathrm{F}_{1}(D$. virilis $\times D$. lummei $)$ males have lost the paternal dot chromosome in the first mitotic cleavage (Sidorova, 1974), they produce nullo-6 sperm also (Evgen'ev \& Sidorova, 1976).

\section{Acknowledgements}

This work was supported by the Natural Science Research Council (Project 1051049). I thank Jaakko Lumme and Allen Orr for comments on the manuscript.

\section{References}

ALEXANDER, M. L. 1976. The genetics of Drosophila virilis. In: Ashburner, M. and Novitski, E. (eds) The Genetics and Biology of Drosophila, vol. 1c, pp. 1365-1427. Academic Press, London.

BARR, A. R. 1980. Cytoplasmic incompatibility in natural populations of a mosquito, Culex pipiens L. Nature, 283, 71-72.

COYNE, J. A. 1985. Genetic studies of three sibling species of Drosophila with relationship to theories of speciation. Genet. Res. Camb., 46, 169-192.

COYNE, J. A. AND ORR, H. A. 1989. Two rules of speciation. In: Otte, D. and Endler, J. A. (eds) Speciation and its Consequences, pp. 180-207. Sinnauer Associates Inc., MA, USA.

DAVIDSON, E. H. 1986. Gene Activity in Early Development. Academic Press, Orlando, FL.

EVGEN'EV, M. B. AND SIDOROVA, N. v. 1976. Genetic regulation of chromosome behaviour in interspecific hybrids of Drosophila. Theor. Appl. Genet., 48, 55-61.

EVGEN'EV, M. B., STEPANOVA, N. G., LEVIN, A. V., SHILOV, A. S. AND TCHERNYSHOV. 1983. The molecular nature of the toromere in Drosophila lummei Hackman and its role in chromosome behavior. Caryologia, 36, 89-99.

HOFFMANN, A. A. AND TURELLI, M. 1988. Unidirectional incompatibility in Drosophila simulans: inheritance, geographic variation and fitness effects. Genetics, 119, 435-444.

HOFFMANN, A. A., TURELli, M. AND SIMMONS, G. M. 1986. Unidirectional incompatibility between populations of Drosophila simulans. Evolution, 40, 692-701.

LAKOVAARA, S. 1969. Malt as a culture medium for Drosophila species. Drosoph. Inf. Serv., 44, 128.

LUMME, J. AND HEIKKINEN, E. 1990. Viability of first and second generation hybrids of Drosophila virilis and Drosophila lummei. Heredity, 65, 435-447.

MITRofanov, v. G. AND SIDOROVA, N. v. 1979. Phenogenetic analysis of chromosome behavior during mitosis in female Drosophila virilis $\times$ male Drosophila lummei hybrids. Genetika, XV, 1221-1227. (In Russian with an abstract in English.)

MITROFANOV, V. G., SIDOROVA, N. V., GRIGORIEVA, G. A. AND POLUEKTOVA, E. V. 1990. Inversion polymorphism in Drosophila lummei Hackman (D. littoralis Sokolov) and its relation to elimination of chromosome 6 of $D$. lummei in the virilis-lummei hybrids. Genetika, 26, 58-64. (In Russian with an English abstract.)

ORR, H. A. 1990. Developmental anomalies in Drosophila hybrids are apparently caused by loss of microchromosome. Heredity, 64, 255-262.

sidorova, N. v. 1974. Changes of the maternal effect in the Drosophila hybrids at the low temperature. Ontogenez, 5, 297-299. (In Russian with an abstract in English.)

SNEDECOR, G. W. AND COCHRAN, w. G. 1967. Statistical Methods. The Iowa State University Press, Ames, IO.

SOKAL, R. R. AND ROHLF, F. J. 1981. Biometry. W. H. Freeman and Company, San Francisco, CA.

sokolov, N. N. 1948. Elimination of chromosomes in interspecific hybrids of Drosophila and the problems of distant hybridization. Lectures from the Academy of Sciences, USSR, 59, 163-166. (In Russian.)

sokolov, N. N. 1959. Cooperation Between Nucleus Cytoplasm and Remote Hybridization of Animals. Publishing House of the Academy of Sciences, USSR, Moscow, USSR. (In Russian.)

StURTEvant, A. H. 1920. Genetic studies on Drosophila simulans. I. Introduction: Hybrids with Drosophila melanogaster. Genetics, 5, 488-500.

THROCKMORTON, L. H. 1982. The virilis species group. In: Ashburner, M., Carson, H. L. and Thompson, J. N. (eds) The Genetics and Biology of Drosophila, vol. 3b, pp. 227-296. Academic Press, London.

WEISBROT, D. R. 1963. Studies on differences in the genetic architecture of related species of Drosophila. Genetics, $\mathbf{4 8}$, 1121-1139. 